NASA/CR-2001-211116

AIAA-2001-3361

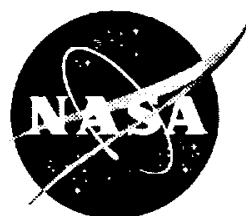

Antimatter Production at a Potential Boundary

Michael R. LaPointe

Ohio Aerospace Institute, Brook Park, Ohio 
Since its founding, NASA has been dedicated to the advancement of aeronautics and space science. The NASA Scientific and Technical Information (STI) Program Office plays a key part in helping NASA maintain this important role.

The NASA STI Program Office is operated by Langley Research Center, the Lead Center for NASA's scientific and technical information. The NASA STI Program Office provides access to the NASA STI Database, the largest collection of aeronautical and space science STI in the world. The Program Office is also NASA's institutional mechanism for disseminating the results of its research and development activities. These results are published by NASA in the NASA STI Report Series, which includes the following report types:

- TECHNICAL PUBLICATION. Reports of completed research or a major significant phase of research that present the results of NASA programs and include extensive data or theoretical analysis. Includes compilations of significant scientific and technical data and information deemed to be of continuing reference value. NASA's counterpart of peerreviewed formal professional papers but has less stringent limitations on manuscript length and extent of graphic presentations.

- TECHNICAL MEMORANDUM. Scientific and technical findings that are preliminary or of specialized interest, e.g., quick release reports, working papers, and bibliographies that contain minimal annotation. Does not contain extensive analysis.

- CONTRACTOR REPORT. Scientific and technical findings by NASA-sponsored contractors and grantees.
- CONFERENCE PUBLICATION. Collected papers from scientific and technical conferences, symposia, seminars, or other meetings sponsored or cosponsored by NASA.

- SPECIAL PUBLICATION. Scientific, technical, or historical information from NASA programs, projects, and missions, often concerned with subjects having substantial public interest.

- TECHNICAL TRANSLATION. Englishlanguage translations of foreign scientific and technical material pertinent to NASA's mission.

Specialized services that complement the STI Program Office's diverse offerings include creating custom thesauri, building customized data bases, organizing and publishing research results ... even providing videos.

For more information about the NASA STI Program Office, see the following:

- Access the NASA STI Program Home Page at http://www.sti.nasa.gov

- E-mail your question via the Internet to help@sti.nasa.gov

- Fax your question to the NASA Access Help Desk at 301-621-0134

- Telephone the NASA Access Help Desk at $301-621-0390$

- Write to: NASA Access Help Desk NASA Center for AeroSpace Information 7121 Standard Drive Hanover, MD 21076 
NASA/CR-2001-211116

AIAA-2001-3361

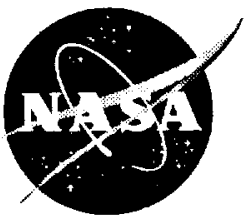

\section{Antimatter Production at a Potential Boundary}

Michael R. LaPointe

Ohio Aerospace Institute, Brook Park, Ohio

Prepared for the

37th Joint Propulsion Conference and Exhibit

cosponsored by the AIAA, ASME, SAE, and ASEE

Salt Lake City, Utah, July $8-11,2001$

Prepared under Cooperative Agreement NCC3-860 and SBIR Contract NAS8-98109

National Aeronautics and

Space Administration

Glenn Research Center 


\section{Acknowledgments}

Funding for this research was provided through NASA SBIR Contract NAS8-98109 during the period April-November 1998.

This report is a formal draft or working paper, intended to solicit comments and ideas from a technical peer group.

This report contains preliminary
findings, subject to revision as
analysis proceeds.

Available from

NASA Center for Aerospace Information

7121 Standard Drive

Hanover, MD 21076
National Technical Information Service 5285 Port Royal Road Springfield, VA 22100 


\title{
ANTIMATTER PRODUCTION AT A POTENTIAL BOUNDARY
}

\author{
Michael R. LaPointe \\ Ohio Aerospace Institute \\ Brook Park, Ohio 44142
}

\begin{abstract}
Current antiproton production techniques rely on highenergy collisions between beam particles and target nuclei to produce particle and antiparticle pairs, but inherently low production and capture efficiencies render these techniques impractical for the cost-effective production of antimatter for space propulsion and other commercial applications. Based on Dirac's theory of the vacuum field, a new antimatter production concept is proposed in which particle-antiparticle pairs are created at the boundary of a steep potential step formed by the suppression of the local vacuum fields. Current antimatter production techniques are reviewed, followed by a description of Dirac's relativistic quantum theory of the vacuum state and corresponding solutions for particle tunneling and reflection from a potential barrier. The use of the Casimir effect to suppress local vacuum fields is presented as a possible technique for generating the sharp potential gradients required for particle-antiparticle pair creation.
\end{abstract}

\section{INTRODUCTION}

Present chemical engines and electric propulsion thrusters are well suited for near-Earth applications and robotic space flight, but advanced propulsion technologies must be developed to enable fast piloted and robotic deep space missions. Of all the known energy sources, none provides more specific energy than the annihilation of matter and antimatter. The energy released per kilogram of combined matter and antimatter is nearly 250 times the specific energy released in nuclear fusion, and over 8 orders of magnitude greater than the specific energy released in chemical combustion. ${ }^{1}$ The possibility of producing photon rockets using gamma rays from electronpositron annihilation was investigated over half a century ago, ${ }^{2}$ but the efficiency of the engines were curtailed by an inability to collimate the energetic photons. With the experimental discovery of the antiproton in 1955, attention turned to the use of protonantiproton annihilation as an energy source for spacecraft propulsion. The higher rest mass energy of the proton-antiproton pair yields $1877 \mathrm{MeV}$ per annihilation event, compared with $1.02 \mathrm{MeV}$ released by electron-positron annihilation. Equally important, a significant fraction of the proton-antiproton annihilation energy appears in the kinetic energy of charged particles, ${ }^{3-5}$ which may be collimated for direct thrust or used to heat an expellant more effectively than electronpositron gamma radiation. Several antiproton-powered rocket designs have been proposed over the past few decades, ranging from low thrust, high specific impulse pion engines to higher thrust, lower specific impulse solid and gas core thermal rockets. ${ }^{6-20}$ Recent modeling efforts have simulated the performance of magnetically confined hydrogen plasma engines heated by charged proton-antiproton annihilation byproduct $\mathrm{s}^{21-24}$ and have investigated antiproton-boosted fission reactions as a driver for an inertial confinement fusion rocket. ${ }^{25.26}$

Although a number of potential antiproton propulsion concepts have been analyzed, their transition from theoretical design to experimental validation and practical use has been constrained by the prohibitive cost of creating and storing the antiprotons. The following section discusses current antiproton production methods, and outlines near-term prospects for efficient antiproton production and storage.

\section{Current Antiproton Production Methods}

The two leading facilities for antiproton production and storage are the European Laboratory for Particle Physics (formerly CERN, the Center for European Nuclear Research) in Geneva, and the Fermi National Accelerator Laboratory (FNAL) in the United States. At the 
CERN facility, protons are accelerated by a linear accelerator to $50 \mathrm{MeV}\left(8 \times 10^{-12} \mathrm{~J}\right)$, injected into a booster ring and accelerated to $800 \mathrm{MeV}$, and then sent to a proton synchrotron, where they are further accelerated to $26 \mathrm{GeV}$. The high-energy protons are then focused into a $2-\mathrm{mm}$ beam and directed into a $3-\mathrm{mm}$ diameter, $11-\mathrm{cm}$ long copper wire target. The relativistic protons collide with the target nuclei, producing a spray of gammas, pions, kaons, and baryons, including antiprotons. On leaving the target, the antiprotons have a peak momentum of $3.5 \mathrm{GeV} / \mathrm{c}$, corresponding to a peak energy of roughly $3 \mathrm{GeV}$. A short focal length, pulsed magnetic hom is used to capture antiprotons that have momenta within $1.5 \%$ of their peak value, at angles up to $50 \mathrm{mrad}$ from the target centerline. The captured antiprotons are sent to a storage ring in bursts of about $10^{7}$ antiprotons every few seconds, and around $10^{11}$ antiprotons can be accumulated before space charge effects scatter the circulating beam. The antiprotons are sent back to the proton synchrotron, which decelerates them to an energy of $200 \mathrm{MeV}$, and then to the low energy antiproton ring, where the circulating beam is further decelerated, stochastically cooled, and stored. Similar techniques are used to create antiprotons at FNAL.

During the high-energy collisions, approximately one antiproton is created for every $10^{5}-10^{6}$ high-energy protons incident on the target. The energy efficiency, defined as the energy released in a proton-antiproton annihilation event ( $1.88 \mathrm{GeV}$ at rest) divided by the energy required to create an antiproton, is abysmally low. On average, CERN creates 1 antiproton for every $2.5 \times 10^{6}$ protons; at an average energy of $26 \mathrm{GeV}$ per proton, the corresponding energy efficiency is approximately $3 \times 10^{-8}$. FNAL, which uses a $120 \mathrm{GeV}$ proton beam to strike the target, creates $I$ antiproton for every $3.3 \times 10^{4}$ protons, corresponding to an energy efficiency of around $4 \times 10^{-7}$. Assuming a "wall-plug" efficiency for each accelerator of around $5 \%$, the total antiproton production efficiencies are roughly $1.5 \times 10^{-9}$ for CERN and $2 \times 10^{-8}$ for FNAL. The total annihilation energy contained in 1-mg of antiprotons (roughly $6 \times 10^{20}$ antiprotons) is $1.8 \times 10^{11} \mathrm{~J}$; an efficiency of $1.5 \times 10^{-9}$ means that it would take nearly $1.2 \times 10^{20} \mathrm{~J}$ $\left(3.3 \times 10^{13} \mathrm{~kW}-\mathrm{hr}\right)$ to create $1 \mathrm{mg}$ of antiprotons. Assuming a conservative energy cost of $\$ 0.05 / \mathrm{kW}-\mathrm{hr}$, the estimated production cost is a staggering $\$ 1.6 \times 10^{11}$ per milligram of antiprotons. Most antimatter propulsion concepts require milligrams to grams of antiprotons, indicating that current antiproton production techniques are inadequate for future spacecraft propulsion applications. However, as discussed by Forward ${ }^{1}$ and Schmidt et al., ${ }^{27}$ neither CERN nor FNAL were designed as dedicated antiproton production facilities. As such, a number of upgrades to the current facilities could be made to improve antiproton production and storage capabilities. Magnetic fields produced by electric currents flowing through the metal wire targets could be used to keep the spray of antiprotons closer to the target axis, reducing their angular spread. Multiple targets could be employed, with magnetic lenses used to refocus the antiprotons between each section. Angular capture efficiencies could be improved by going to higher beam energies, creating a forward-peaked distribution that allows more antiprotons to be captured. Material lenses could be replaced with current-canrying plasma lenses, which are less likely to absorb the antiprotons and would not need active cooling. Using linear rather than synchrotron accelerators to produce the initial high-energy proton beams could increase the accelerator energy efficiency by an order of magnitude over the current $5 \%$ wall plug efficiencies.

Taken together, the potential facility improvements could result in the yearly production and storage of microgram quantities of antiprotons at a potential $\operatorname{cost}^{27}$ of around $\$ 6.4 \times 10^{6} / \mu \mathrm{g}\left(\$ 6.4 \times 10^{9} / \mathrm{mg}\right)$. While these production numbers and costs are approaching those required for ground testing antimatter propulsion concepts, they are not adequate for antimatter-based propulsion systems. Forward ${ }^{1.20}$ calculates that antiproton propulsion becomes cost competitive with chemical propellant systems at an antiproton production cost of approximately $\$ 10^{7} / \mathrm{mg}$, and antiproton propulsion becomes the most cost effective propulsion source available if the production costs can be lowered to $\$ 2 \times 10^{6} / \mathrm{mg}$. Because the near term facility modifications outlined above are unlikely to produce the necessary reduction in antiproton production costs, a number of alternative antiproton production techniques have been suggested. Chapline ${ }^{28}$ has proposed colliding heavy ion beams, made up of singly charged uranium atoms, to produce up to $10^{18}$ antiprotons/sec. Unfortunately, the antiprotons will be emitted isotropically and will be very difficult to collect. Equally problematic, the colliding heavy ion beams will produce a significant amount of nuclear debris and radiation, which would have to be safely and efficiently removed from the spray of antiprotons. Cassenti ${ }^{29}$ has suggested that the pions generated during the collision of high-energy protons with heavy target nuclei could be redirected toward the target to increase the number of antiprotons and improve the efficiency of current antiproton production techniques. Although promising, the collection and redirection of the pions and antiprotons remains a major challenge to this concept.

Hora ${ }^{30}$ proposed the use of a high intensity laser that could generate sufficiently strong electric fields to 
produce proton-antiproton pairs from the vacuum, and Crowe $^{31}$ separately proposed the use of high intensity lasers to produce electron-positron pairs. At present, however, there are no known lasers that can produce the high intensity electric fields needed for pair production. Forward $^{1.20}$ and Haloulakos and Ayotte ${ }^{32}$ have investigated the possibility of building and operating an antiproton factory in space, where the proton accelerator could be powered by solar energy. However, the estimated cost to produce and store the antiprotons is still nearly $\$ 10^{9} / \mathrm{mg}$, which is a factor of $10^{2}$ too high for cost-effective space propulsion applications. ${ }^{32}$

Rather than rely on high-energy proton beam collisions with a stationary target, this paper outlines a new concept that may lead to the more efficient production of antimatter in quantities sufficient for propulsion and other commercial applications. The proposed technique is based upon particle-antiparticle pair production at the steep potential boundary created by the suppression of local vacuum field energies. The premise is based on Dirac's relativistic theory of the vacuum state, which is outlined in the following section. The theory underlying particle-antiparticle pair creation at a potential boundary is discussed, followed by an explanation of the technique proposed to create the required potential step. The paper concludes with an overview of an experimental approach designed to demonstrate the feasibility of this new antimatter production concept.

\section{DIRAC'S THEORY OF THE VACUUM STATE}

Dirac was the first to develop a relativistic wave equation that correctly describes the interaction of spin-1/2 particles, such as electrons and protons. ${ }^{33}$ Dirac's equation contains both positive and negative energy solutions, the latter identified with the continuum energy of the vacuum state (Fig. 1). As defined by Dirac, the vacuum state is characterized by the absence of all real electrons in positive energy states, but has electrons filling all negative energy states (the "Dirac sea"). Because of the Pauli exclusion principle, real electrons cannot transition into negative energy states since all such states are already occupied; however, an electron in a negative energy state can absorb radiation and transition to a positive energy state, leaving behind a "hole" in the negative energy continuum. The hole behaves like a positive electron and represents the antiparticle of the electron. The creation of an electron and an antielectron (positron) is identified as pair creation and requires a minimum energy of $2 \mathrm{~m}_{0} \mathrm{c}^{2}$. Pair annihilation occurs when an electron drops back into the (unoccupied) hole, with the resulting transition energy emitted as radiation.

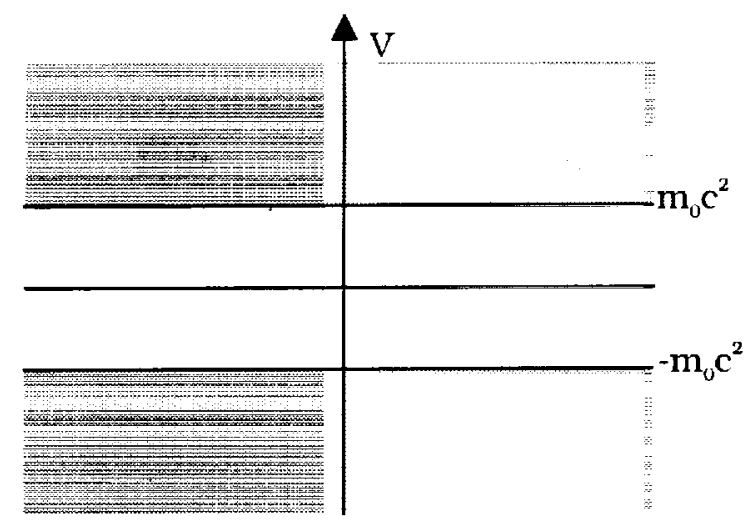

Figure 1. Energy Levels of the Dirac Equation

More generally, in Dirac's theory the vacuum represents a continuum of negative energy states occupied by negative energy particles. Pair creation is the process in which sufficient energy is given to a particle in the negative energy state to raise it to a positive energy state (creating a real particle and leaving behind a hole, or antiparticle); annihilation occurs when the particle falls back into the hole, with the energy carried away as radiation. The vacuum itself should have zero energy, zero mass, and no charge, which is clearly not satisfied by the simple form of the theory. Instead, there are infinitely many negative energy states, which together have an infinitely large negative energy, and, in the case of electrons populating the negative energy continuum, an infinitely large negative charge. These difficulties are removed by renormalizing the zero point of charge and energy in such a way that the vacuum has no mass, energy, or charge. This renormalization process is not pleasing from an aesthetic viewpoint, but it does satisfy the constraint that only departures from the vacuum state are observable and hence relevant.

\section{TUNNELING AND POTENTIAL BARRIERS}

Related to Dirac's theory of the vacuum is the quantum mechanical process of particle tunneling in the presence of a steep potential step. An overview of this process is provided by Greiner, ${ }^{34}$ the salient features of which are given here.

Consider a spin-1/2 particle (for example, an electron or proton) with energy, E, and momentum, p, traveling along the $z$-axis (Figure 2). The particle encounters a step potential of magnitude $V_{0}$ that rises to full value in a distance equal to the Compton wavelength of the particle, $\lambda_{\mathrm{c}}$ :

$$
\lambda_{c}=\frac{h}{m_{0} c}
$$


where $h$ is Planck's constant $\left(6.626 \times 10^{-34} \mathrm{~J}\right.$-s $), m_{0}$ is the particle rest mass, and $c$ is the speed of light.

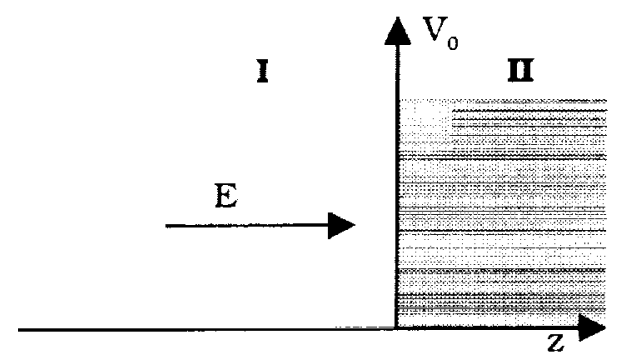

Figure 2. Particle incident on a potential step.

The Dirac equation describing the propagation of the particle in Region I is:

$i \hbar \frac{\partial \psi}{\partial t}=\left[\frac{\hbar c}{i}\left(\hat{\alpha}_{1} \frac{\partial}{\partial x^{1}}+\hat{\alpha}_{2} \frac{\partial}{\partial x^{2}}+\hat{\alpha}_{3} \frac{\partial}{\partial x^{3}}\right)+\hat{\beta}_{m_{0}} c^{2}\right] \psi=H_{f} \psi$

where $\psi$ is the particle wave function, $\hbar$ is the reduced Planck constant ( $\mathrm{h} / 2 \pi), H_{f}$ is the Hamiltonian, and $\hat{\alpha}, \hat{\beta}$ are the standard Dirac matrices. Noting that the momentum operator $p$ is given by:

$$
\vec{p}=\frac{\hbar c}{i}\left(\hat{\alpha}_{1} \frac{\partial}{\partial x^{1}}+\hat{\alpha}_{2} \frac{\partial}{\partial x^{2}}+\hat{\alpha}_{3} \frac{\partial}{\partial x^{3}}\right)=\frac{h}{i} \nabla
$$

the Dirac equation can be written in the more compact form:

$$
i \hbar \frac{\partial \psi}{\partial t}=\left[c(\hat{\alpha} \cdot \dot{p})+\hat{\beta} m_{0} c^{2}\right] \psi=H_{f} \psi
$$

The Hamiltonian for Region I (zero potential) is the total particle energy, E, while in Region II the Hamiltonian becomes $\left(E-V_{0}\right)$. The Dirac equation for a particle wave traveling along the $+z$ direction in Region $I$ is then:

$$
\left[c\left(\hat{\alpha}_{3} \cdot \vec{p}_{1}\right)+\hat{\beta} m_{0} c^{2}\right] \psi=E \psi
$$

In Region II, the Dirac equation for the traveling particle wave becomes:

$$
\left[c\left(\hat{\alpha}_{3} \cdot \vec{p}_{1}\right)+\hat{\beta} m_{0} c^{2}\right] \psi=\left(E-V_{0}\right) \psi
$$

The solution for the particle wave function in Region I is:

$$
\psi_{I}=A\left(\begin{array}{c}
1 \\
0 \\
\frac{p_{1} c}{E+m_{0} c^{2}} \\
0
\end{array}\right) \exp \left[\frac{i p_{1} z}{\hbar}\right]
$$

where $A$ is a constant and the particle momentum, $p_{1}$, is given by:

$$
p_{1} c=\sqrt{E^{2}-m_{0}^{2} c^{4}}
$$

At the potential boundary, part of the particle wave will be reflected and part will be transmitted. The reflected wave solution in Region I is:

$$
\psi_{t}^{r}=B\left(\begin{array}{c}
1 \\
0 \\
\frac{-p_{1} c}{E+m_{0} c^{2}} \\
0
\end{array}\right) \exp \left[\frac{-i p_{1}-}{h}\right]
$$

and the transmitted solution in Region II is:

$$
\psi_{n}=D\left(\begin{array}{c}
1 \\
0 \\
\frac{-p_{2} c}{V_{0}-E-m_{0} c^{2}}
\end{array}\right) \exp \left[\frac{i p_{2} z}{\hbar}\right]
$$

where again $\mathrm{B}$ and $\mathrm{D}$ are constants. The particle momentum in Region II is given by:

$$
p_{2} c=\sqrt{\left(E-V_{0}\right)^{2}-m_{0}^{2} c^{4}}
$$

The incident and reflected wave functions must equal the transmitted wave function at the step boundary $(\mathrm{z}=0)$ :

$$
\left.\psi_{I}\right|_{i=0}+\left.\psi_{l}^{r}\right|_{:=0}=\left.\psi_{I I}\right|_{:=0}
$$

from which the following conditions are obtained for the coefficients A, B and D:

$$
A+B=D
$$

$$
\begin{aligned}
A-B & =-D \frac{p_{2}}{p_{1}}\left(\frac{E+m_{0} c^{2}}{V_{0}-E-m_{0} c^{2}}\right) \\
& =-D \sqrt{\frac{\left(V_{0}-E+m_{0} c^{2}\right)\left(E+m_{0} c^{2}\right)}{\left(V_{0}-E-m_{0} c^{2}\right)\left(E-m_{0} c^{2}\right)}}=-D \gamma
\end{aligned}
$$

where $\gamma$ is defined as:

$$
\gamma=\sqrt{\frac{\left(V_{0}-E+m_{0} c^{2}\right)\left(E+m_{0} c^{2}\right)}{\left(V_{0}-E-m_{0} c^{2}\right)\left(E-m_{0} c^{2}\right)}}
$$


Upon rearrangement, Equations 13 and 14 yield:

$$
\frac{B}{A}=\left(\frac{1-\gamma}{1+\gamma}\right)
$$

Dividing Equation 14 by the coefficient $A$ and substituting Equation 16 for $(B / A)$ yields:

$$
\frac{D}{A}=\frac{2}{1-\gamma}
$$

The particle current $j$ is defined to be:

$$
j(z)=c \psi^{+}(z) \hat{\alpha} \psi(z)
$$

where $\psi^{\dagger}(z)$ is the adjoint of $\psi(\xi)$. The values of $\psi^{\dagger} \hat{\alpha}$ in Region I are:

$$
\begin{aligned}
& \psi_{l}^{\dagger} \hat{\alpha}_{1}=A^{*}\left(0, \frac{p_{1} c}{E+m_{0} c^{2}}, 0,1\right) \exp \left[\frac{-i p_{1} z}{\hbar}\right] \\
& \psi_{I}^{\dagger} \hat{\alpha}_{2}=A^{*}\left(0,-i \frac{p_{2} c}{E+m_{0} c^{2}}, 0,-i\right) \exp \left[\frac{-i p_{1} z}{h}\right] \\
& \psi_{I}^{\dagger} \hat{\alpha}_{3}=A^{*}\left(\frac{p_{1} c}{E+m_{0} c^{2}}, 0,1,0\right) \exp \left[\frac{-i p_{1} z}{h}\right]
\end{aligned}
$$

from which $j_{1}$, the incident particle current in Region I, is:

$$
j_{1}=-A A^{*} \frac{2 p_{1} c^{2}}{E+m_{0} c^{2}}
$$

Similarly, the reflected $\left(\mathrm{j}_{1}^{\mathrm{r}}\right)$ and transmitted $\left(\mathrm{j}_{\mathrm{II}}\right)$ particle currents are:

$$
\begin{aligned}
& j_{l}^{r}=-B B^{*} \frac{2 p_{1} c^{2}}{E+m_{0} c^{2}} \\
& j_{l l}=D D^{*}\left(\frac{-2 p_{1} c^{2}}{V_{0}-E-m_{0} c^{2}}\right)
\end{aligned}
$$

Equations 20-22 can now be used to calculate the reflection and transmission coefficients for the particle wave function impacting the potential boundary. Taking the ratio of the reflected current to the incident particle current yields:

$$
\frac{\left|j_{I}^{r}\right|}{\left|j_{l}\right|}=\left|\frac{B B^{*} \frac{2 p_{1} c^{2}}{E+m_{0} c^{2}}}{A A^{*} \frac{2 p_{1} c^{2}}{E+m_{0} c^{2}}}\right|=\left|\frac{B B^{*}}{A A^{*}}\right|=\left|\frac{B^{2}}{A^{2}}\right|=\frac{(1+\gamma)^{2}}{(1-\gamma)^{2}}
$$

The ratio of the transmitted current to the incident current is given by:

$$
\begin{aligned}
\frac{\left|j_{n}\right|}{\left|j_{l}\right|} & =\left|\frac{D D^{*} \frac{2 p_{2} c^{2}}{V_{0}-E-m_{0} c^{2}}}{A A^{*} \frac{2 p_{1} c^{2}}{E+m_{0} c^{2}}}\right|=\left|\frac{D D^{*}}{A A^{*}}\right| \gamma \\
& =\left|\frac{D^{2}}{A^{2}}\right| \gamma=\frac{4 \gamma}{(1-\gamma)^{2}}
\end{aligned}
$$

For a potential step $\mathrm{V}_{0}>\left(E+\mathrm{m}_{0} \mathrm{c}^{2}\right)$, the value $\gamma>\mathbf{l}$. From Equation 23, this indicates that the reflected particle current exceeds the incident particle current in Region I $\left(\mathbf{j}_{l}{ }_{l}|>| \mathrm{j}_{1} \mid\right)$. It appears that electrons are entering Region I from Region II, but there are no electrons initially present in Region II. This result, known as Klein's Paradox, is most often interpreted as particleantiparticle pair creation at the potential boundary.

\section{Discussion of Results.}

Applying a potential $\mathrm{V}_{0}>\mathrm{E}+\mathrm{m}_{0} \mathrm{c}^{2}$ raises the energy in Region II sufficiently for there to be an overlap between the negative energy continuum $(z>0)$ and the positive energy continuum $(z<0)$, as shown in Figure 3:

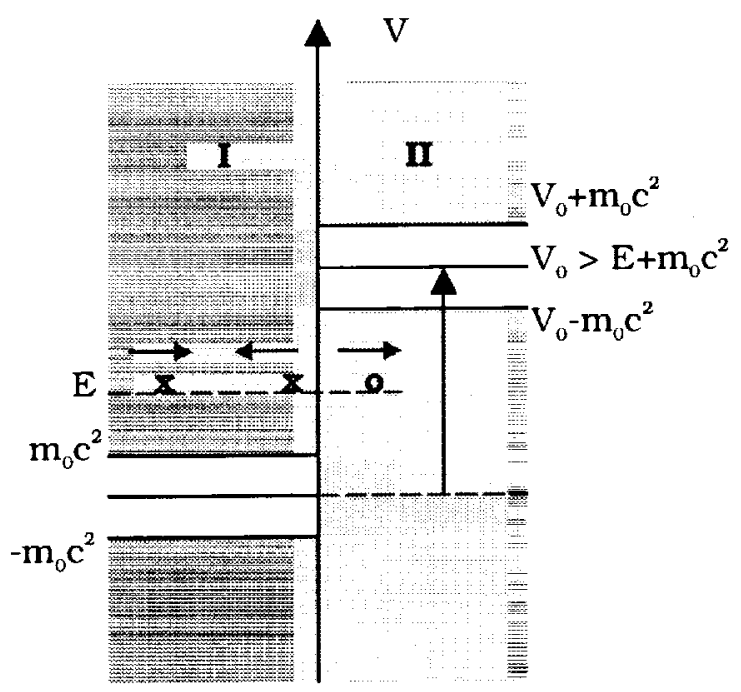

Figure 3. Energy Continuum of the Dirac Equation at a Potential Barrier. ${ }^{34}(\mathrm{x})=$ particle, $(0)=$ antiparticle

When $\mathrm{V}_{0}>\mathrm{E}+\mathrm{m}_{0} \mathrm{c}^{2}$, the particles striking the barrier from the left are able to knock additional particles out of the vacuum on the right, leading to an antiparticle current flowing from the left to the right in Region II and a particle current flowing from right to left in Region I. This pair creation is depicted schematically in Figure 3, with the additional particles entering Region I from the right accounting for the increase in 
return current. As noted by Greiner, ${ }^{34}$ this process is most readily understood as particle-antiparticle pair creation at the potential barrier and is related to the decay of the vacuum in the presence of supercritical fields. When the potential function $V_{0}$ is less than $E+$ $\mathrm{m}_{0} \mathrm{c}^{2}$, the particle momentum in Region II is imaginary (Eq. 11) and the wave solution will be exponentially damped (Eq. 10); all of the incident current is then reflected back into Region $I$, and no particle current is transmitted into Region II. It is only when $V_{0}>E+$ $\mathrm{m}_{0} \mathrm{c}^{2}$ that the momentum in Region II becomes real and the particle wave function in Region II again becomes a traveling wave.

The above derivations may be summarized as follows:

- The Dirac equation is used to represent the evolution of spin-1/2 particle wave functions.

- Dirac's equation permits both positive and negative energy solutions; the negative energy states are filled with virtual particles, which prevent particle transitions from positive energy states to negative energy states via the Pauli exclusion principle.

- Particles will be completely reflected from a potential barrier $\left(V_{0}\right)$ when their energy $E<V_{0}$; the transmitted particle wavefunction is exponentially damped within the potential barrier.

- For potential steps with $\mathrm{V}_{0}>\mathrm{E}+\mathrm{m}_{0} \mathrm{c}^{2}$, an incident particle will induce pair creation at the potential boundary, resulting in a (real) return particle current and a (real) transmitted antiparticle current.

The question now arises as to whether this effect can actually be applied to the production of antimatter. The potential step must be greater than $\mathrm{E}+\mathrm{m}_{0} \mathrm{c}^{2}$, where $\mathrm{E}$ is the total particle energy (rest mass plus kinetic), and the potential must rise to its full value over a distance comparable to the Compton wavelength of the particle, $\mathrm{h} /\left(\mathrm{m}_{0} \mathrm{c}\right)$. For an electron, the minimum potential step height is $1.02 \mathrm{MeV}$ (plus the kinetic energy of the electron), and the Compton wavelength is approximately $2.4 \times 10^{-12} \mathrm{~m}$. For a proton, the minimum potential step height is $1876 \mathrm{MeV}$ and the Compton wavelength is approximately $1.3 \times 10^{-16} \mathrm{~m}$. These supercritical potentials are too large to be generated over such short distances using laboratory electric fields, but there may be another option: rather than use externally applied fields to raise the vacuum energy in Region II, it may be possible to use the Casimir effect to lower the vacuum energy in Region I. This concept, shown schematically in Figure 4, may be able to produce the same pair creation effects depicted in Figure 3 without requiring the application of supercritical external fields. A brief discussion of the Casimir effect is provided below, followed by an outline of a possible experimental test of the proposed antimatter production mechanism.

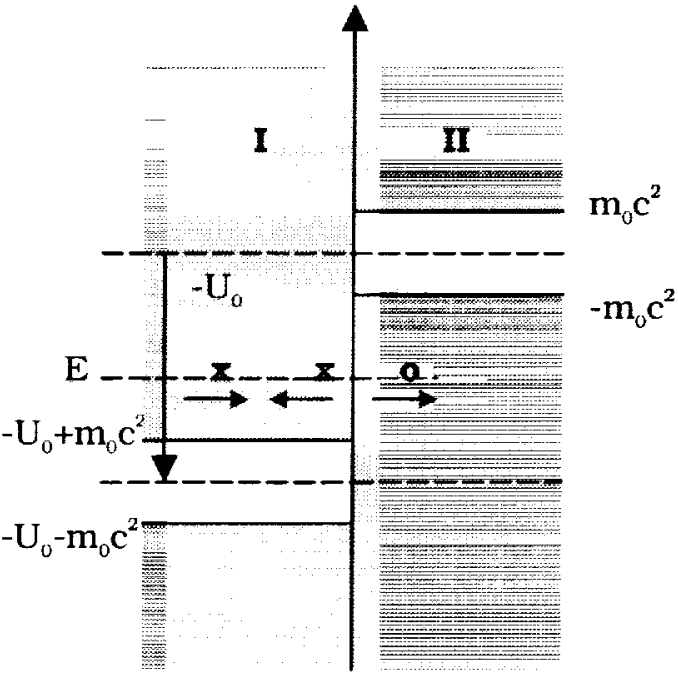

Figure 4. Lowering the vacuum energy via the Casimir effect.

\section{VACUUM FIELDS AND THE CASIMIR EFFECT}

Dirac's vacuum is a negative energy continuum populated by particles that prevent positive energy particles from transitioning into the negative energy states. To avoid obvious problems associated with infinite vacuum charges and energies, the vacuum state is renormalized to zero; only deviations from the vacuum state are measurable. As such, the potential step shown in Figure 3 represents an applied field measured with respect to the background vacuum; the energy in Region II has been raised by the applied field such that the negative energy states now overlap the positive energy states in Region I. However, raising the background vacuum in Region II requires a tremendous amount of energy $\left(V_{0}>E+m_{0} c^{2}\right)$ over a very small distance, which is clearly beyond present capabilities. Rather than raising the vacuum energy in Region II, it is proposed that the same effect can be generated by suppressing the relative vacuum energy in Region I, as shown in Figure 4. This process can be viewed either as lowering the positive energy states such that they now overlap with the negative energy states in Region II, or the back. ground vacuum in Region I can be renormalized to zero to yield an energy diagram similar to Figure 3 . In either instance, a particle wave traveling from Region I into Region II will be described by the same solutions outlined in the section above, leading to particleantiparticle pair creation at the potential boundary. Unlike Figure 3, pair production is achieved not by raising the potential but by lowering the relative vacuum energy, an effect that might be accomplished through the use of a Casimir cavity. 
Before discussing the Casimir effect, it should be noted that Dirac's interpretation of the vacuum as a continuum of negative energy states occupied by negative energy particles, though somewhat dated, is not in disagreement with the current quantum electrodynamic (QED) interpretation of the vacuum as an infinite sea of electromagnetic radiation populated with virtual particle pairs. Because exchange interactions occur in Dirac's theory, virtual electron-positron pairs are continuously created and annihilated in the vacuum; an electron in a bound or free state can fill a virtual hole in the Dirac sea, with a virtual electron taking its place. Renormalization of the vacuum energy and charge is required both in Dirac's original theory and in QED, and as noted by Greiner, ${ }^{34}$ the physical content of the Dirac theory forms the basis of current quantum electrodynamics. This is mentioned because the Casimir effect is generally discussed in terms of the QED interpretation of the vacuum state, and it is necessary to point out that both the Dirac and QED vacuum interpretations are complementary.

\section{The Casimir Effect}

As in the Dirac theory, the vacuum state in quantum electrodynamics is interpreted to be the state of lowest energy. This lowest energy state is not at rest, but fluctuates with a "zero-point" energy. ${ }^{35-39}$ The vacuum fluctuations have measurable effects, including the experimentally observed Lamb-Retherford shift between the $s$ and $p$ energy levels of the hydrogen atom, and the attractive Casimir force that occurs between closely spaced uncharged conductors. As discussed by Mostepanenko et al., ${ }^{35}$ the Casimir effect can be accounted for by assuming the force is a consequence of the separation-dependent vacuum field energy trapped between the conductors. For example, assume that two square conducting plates with side dimensions $L$, separated by a distance $z$, are placed in a vacuum. In the QED interpretation, the vacuum is teeming with electromagnetic radiation (although mathematically the vacuum state is renormalized to zero), hence the plates may be considered to constitute a cavity that supports vacuum fluctuation modes with wave numbers down to about $z^{-1}$. The vacuum energy trapped between the plates is approximately given by:

$$
\begin{aligned}
U=\sum_{k=\frac{1}{2}}^{K} \frac{1}{2} h \omega \approx\left(L^{2} z\right) \int_{=-1}^{K} \hbar c k k^{2} d k & \cong \frac{1}{4} L^{2} \hbar c\left[z K^{4}-z^{-3}\right] \\
& =U_{U}-U_{L}
\end{aligned}
$$

where $U_{U}$ is the upper energy bound, $U_{L}$ is the lower energy bound, and $K$ represents a high frequency cutoff to make the total energy finite. The negative rate of change of the lower cut-off energy $U_{L}$ with separation z constitutes a force of attraction, $F$, per unit area, given by:

$$
\frac{F}{A}=-\frac{1}{L^{2}} \frac{d U_{L}}{d z} \approx \frac{\hbar c}{z^{4}}
$$

A more careful analysis leads to the exact relationship for the force per unit area between the parallel conducting plates: ${ }^{39}$

$$
\frac{F}{A}=\frac{\pi^{2}}{240} \frac{h c}{z^{4}}
$$

In other words, the vacuum energy density between the plates is lower than the vacuum energy density outside the plates by an amount equal to the right hand side of Equation 27. For reasonable plate sizes and small separation distances, the change in the vacuum energy density can be appreciable. Assuming the plates have length $L=0.1 \mathrm{~m}$ and are separated by a distance of $1-\mu \mathrm{m}$ $\left(10^{-6} \mathrm{~m}\right)$, the change in the vacuum energy density is approximately $1.3 \times 10^{-4} \mathrm{~J} / \mathrm{m}^{3}$, corresponding to an inward pressure of $1.3 \times 10^{-4} \mathrm{~N} / \mathrm{m}^{2}$ on the plates. For a separation distance of $0.1-\mu \mathrm{m}\left(10^{-7} \mathrm{~m}\right)$, the change in the vacuum energy density is equal to $1.3 \mathrm{~J} / \mathrm{m}^{3}$, corresponding to an inward pressure of $1.3 \mathrm{~N} / \mathrm{m}^{2}$. The calculations can be carried down to separation distances that are approximately equal to the cut-off wavelength of the conducting material, at which point the plates can no longer be considered good electromagnetic reflectors.

Equations 26 and 27 demonstrate that the vacuum energy density between the conducting plates is lower than the external vacuum energy density. Multiplying Equation 27 by the plate surface area $\left(L^{2}\right)$ and the separation distance between the plates $(\xi)$ yields an expression relating the decrease in the vacuum energy between the plates compared to the external vacuum field energy:

$$
\Delta E_{V A C}=-\frac{\pi^{2}}{720} \frac{\hbar c L^{2}}{z^{3}}
$$

For square plate dimensions of $\mathrm{L}=0.1 \mathrm{~m}$ and a separation distance of $0.1-\mu \mathrm{m}$, the change in vacuum energy is calculated to be $4.3 \times 10^{-9} \mathrm{~J}$, or roughly $2.7 \times 10^{10} \mathrm{eV}$ (27 $\mathrm{GeV}$ ). The vacuum energy between the plates is thus substantially lower than the vacuum energy external to the plates, or conversely, if the vacuum energy between the plates is renormalized to zero, the vacuum energy external to the plates is substantially higher than the vacuum energy between the plates. By adjusting the plate dimensions and separation distance, it may thus be possible to significantly suppress the vacuum energy in a given region and generate a condition similar to that shown in Figure 4. A particle generated in the suppressed vacuum fields of Region I will see a higher vacuum field energy outside of the plates, and if the relative change in the vacuum energy exceeds $E+m_{0} c^{2}$, 
it may be possible to generate particle-antiparticle pairs at the vacuum energy step. Because the Dirac solutions hold equally well for electrons or protons, the possibility exists that low energy proton-antiproton pairs might be created at the steep potential boundary created by vacuum field suppression in a Casimir cavity.

\section{Creating a Potential Gradient}

The Casimir effect provides an avenue for creating sufficiently large potential steps, but the question arises as to whether these steps can be generated over a distance comparable to the Compton wavelength of the particle. From Equation 1, the Compton wavelength for electrons is $2.42 \times 10^{-12} \mathrm{~m}$, while for protons the Compton wavelength is $1.32 \times 10^{-15} \mathrm{~m}$. Either distance is several orders of magnitude smaller than any realistic plate thickness separating the interior and exterior vacuum fields, hence typical flat plates will not provide a sufficiently steep potential step for pair creation to occur.

A possible solution to this dilemma is to create a suitable potential gradient over a distance larger than the Compton wavelength, such that the required step change in potential occurs over a distance comparable to the Compton wavelength. Assuming the required potential step has magnitude $V$ and the change in vacuum field energy due to the Casimir effect has magnitude $\Delta E_{\text {vac }}$, the gradient relation can be expressed:

$$
\frac{V}{\lambda_{r}} \approx \frac{\Delta E_{\mathrm{var}}}{d}
$$

where $\lambda_{c}$ is the Compton wavelength of the particle and $d$ is the plate thickness, or more properly the thickness of the region over which the change in vacuum field energy occurs. For pair production to occur at the step boundary, the potential $V$ must exceed $\mathrm{E}+\mathrm{m}_{0} \mathrm{c}^{2}$, where $\mathrm{E}$ is the total energy of the particle (rest mass plus kinetic energy). Assuming the particle kinetic energy at the boundary is small compared to its rest mass energy, the requirement for $\mathrm{V}$ becomes:

$$
V \geq 2 m_{0} c^{2}
$$

Inserting Equations 28 and 30 into Equation 29 yields the following expression for the plate area, thickness, and separation as a function of particle mass and Compton wavelength:

$$
\frac{2 m_{0} c^{2}}{\lambda_{c}}=\frac{\pi^{2} \hbar c L^{2}}{720 z^{3} d}
$$

which reduces to:

$$
\frac{L^{2}}{z^{3} d} \approx 4.15 \times 10^{44} \frac{m_{0}}{\lambda_{c}}
$$

Equation 32 expresses necessary conditions for a flat parallel plate geometry to form a sufficiently steep potential gradient for particle-antiparticle pair production to occur. For electrons, this condition can be written:

$$
\frac{L^{2}}{z^{3} d} \approx 1.56 \times 10^{26}\left(\mathrm{~m}^{-2}\right)
$$

Assuming a plate separation distance $z=10^{-7} \mathrm{~m}$ and a gradient length $d=10^{-6} \mathrm{~m}$, the required plate area, $L^{2}$, is around $0.156 \mathrm{~m}^{2}$; for square plates this corresponds to a side length of approximately $0.4 \mathrm{~m}$. Plates of this size and flatness are well within current manufacturing capabilities, offering some encouragement for experimental verification of the proposed concept. For protons,

$$
\frac{L^{2}}{z^{3} d} \approx 5.26 \times 10^{32}\left(m^{-2}\right)
$$

Using the same plate separation and gradient distances above would require a plate length of $725 \mathrm{~m}$ to provide a suitable potential gradient. However, a number of Casimir force experiments have been performed over the past several years with plate separations down to several nanometers, and a reasonable lower limit of $10^{-8}$ $\mathrm{m}$ can be assumed for the plate separation $z$. The distance over which the potential gradient is formed, $d$, can also be reduced by locally thinning the plate support structure; a realistic lower bound on $d$ is thus assumed to be $10^{-9} \mathrm{~m}$. Given these values, the plate size for producing the required potential step for protonantiproton pair production is around $0.73 \mathrm{~m}$, which is difficult but not impossible to manufacture.

Ideally, the potential gradient would be formed in a region devoid of plate material; this can be accomplished by designing the Casimir plate with a small hole whose diameter is of the same order or smaller than the plate separation distance. Vacuum electromagnetic fields with wavelengths larger than the hole diameter will still be blocked by the cavity, but the potential gradient formed by the plates is now in a material-free region that more faithfully reproduces the assumptions behind Figures 3 and 4 . Possible effects due to the fringing of vacuum electromagnetic fields at the hole boundaries remain to be evaluated, but this method appears promising to provide the required potential gradients in a material-free region.

Summary. In summary, it appears possible to produce a Casimir cavity geometry that will provide sufficiently steep potential gradients for particle-antiparticle pair creation. By introducing a small hole in the plate material with a diameter similar to the plate separation distance, a potential step may be created in a materialfree region, as depicted in Figure 4. Electron-positron pairs and proton-antiproton pairs can conceivably be 
generated using this technique, with significantly less infrastructure and presumably lower cost than current antimatter production methods.

\section{EXPERIMENTAL DESIGN}

The following experiment is proposed to evaluate the possibility of producing particle-antiparticle pairs at a potential boundary created within a Casimir cavity. The proposed experiment is a modified version of a similar experiment performed by the author under a prior contract to the NASA Marshall Space Flight Center. ${ }^{40}$ In that experiment, flat parallel plates were used to form a Casimir cavity to investigate pair creation, but the plate geometries were not properly designed to generate sharp potential gradients. Based on that effort and the additional analysis in this report, the following experiment is suggested as a proof-of-concept test.

\section{Electron-Positron Production}

To investigate the formation of electron-positron pairs, it is proposed that a Casimir cavity be constructed from two flat, square metallic plates, each with an area of $1.61 \times 10^{-2} \mathrm{~m}^{2}(L=12.7 \mathrm{~cm})$. From Equation 33, spacing the plates a distance $z=10^{-7} \mathrm{~m}$ apart should provide a suitably steep potential gradient for electron-positron pair creation to occur. To create a material free region for the steep potential step, the central region of one plate should be thinned down to a material depth $d \leq$ $10^{-7} \mathrm{~m}$, into which a central hole should be drilled perpendicular to the plate boundary. The diameter of the hole should be on the order of the plate separation distance, so that vacuum electromagnetic fields with wavelengths larger than the hole diameter will still be blocked by the cavity plates. On the opposite plate, a small amount of radioactive material can be deposited to act as a source of electrons within the cavity; $\mathrm{Ni}^{63}$ is a readily available commercial source, and the kinetic energy of the emitted electrons $(0.067 \mathrm{MeV})$ are sufficiently below the rest mass energy of the electron that Equation 30 remains a viable approximation. Alternatively, the metallic plate can be irradiated to produce subsequent electron emissions within the cavity.

The plate surfaces must be aligned and moved to within $10^{-7} \mathrm{~m}$ to form the required potential step, which can be accomplished using commercially available piezoelectric transducers. The close separation distance requires that the surface flatness of the plates not exceed $10^{-8} \mathrm{~m}$, which is a stringent but commercially attainable constraint. The entire system should be mounted in a vacuum system capable of achieving a hard vacuum $\left(\leq 10^{-8}\right.$ torr), and isolated from vibrations. To evaluate whether the proposed pair production method works, a small target placed outside the central plate hole can be used to intercept any positrons emitted at the potential boundary. The resulting annihilation of the positrons with the target material will produce $0.511-\mathrm{MeV}$ gamma rays that can be measured with a detector located behind the target.

\section{Proton-Antiproton Production}

If successful, the experimental arrangement outlined above will demonstrate the basic feasibility of the proposed pair production process. However, for propulsion applications it is desirable to produce antiprotons rather than positrons. As previously discussed, the constraints on plate size, flatness, and separation become significantly more demanding, but remain within the capability of current manufacturing techniques. For proton-antiproton pair production, square plates with areas of $0.526 \mathrm{~m}^{2}(L=0.725 \mathrm{~m})$ would have to be separated by a distance of $10^{-8} \mathrm{~m}$, indicating that the surface flatness of the plates would have to be on the order of nanometers. Larger plate areas would relax this constraint by allowing larger separation distances, and the ability to machine flat surface areas must be traded against the fabrication of larger plate dimensions. The central hole diameter would again be on the order of the plate separation distance to provide a material-free potential step, and the electron source would be replaced with a proton source to provide the particles within the cavity. If this scheme is successful, the antiprotons can be captured upon exiting through the hole in the Casimir plate and stored in portable Penning traps for later use.

\section{CONCLUDING REMARKS}

A new concept has been described for creating matterantimatter particle pairs at a steep potential boundary. The potential step is created using the Casimir effect to suppress the vacuum energy between parallel conducting plates. Preliminary calculations indicate that a sufficiently steep potential gradient can be formed for reasonable plate dimensions and separation distances. A preliminary experimental design is outlined as a proofof-concept test for the proposed antimatter production scheme. Additional analysis remains to be performed to validate the concept, including an evaluation of material and temperature effects on the plate boundaries, the effect of fringing fields on the potential step at the plate central hole, and the consequences of imperfect parallel plate alignment on the required potential gradient. Nevertheless, based on the preliminary analysis presented in this paper, the proposed concept appears to be a potentially viable alternative to the high-energy antimatter production methods currently in use. 


\section{REFERENCES}

1. Forward, R.L., Antiproton Amnihilation Propulsion, AFRPL-TR-85-034, Forward Unlimited, Oxnard, CA, 1985.

2. Sanger, E., "Zur Theorie der Photoneraketen" ("The Theory of Photon Rockets"), Ingenieur-Archiv., 21, 1953, pp. 213-226.

3. Morgan, D.L. and Hughes, V.M., "Atomic Processes Involved in Matter-Antimatter Annihilation," Phys. Rev., D-2 (8), 1979, pp. 1389-1391.

4. Agnew, L.E., Jr., Elioff, T., Fowler, W.G., Lander, R.L., Powell, W.M., Segre, E., Steiner, H.M., White, H.S., Wiegand, C., and Ypsilantis, T., "Antiproton Interactions in Hydrogen and Carbon Below $200 \mathrm{MeV}$," Phys. Rer., 118 (5), 1960, pp. 1371-1391.

5. Morgan, D.L., Amnihilation of Antiprotons in Heary Nuclei, AFRPL TR-86-011, Lawrence Livermore National Laboratory, Livermore, CA, Apr 1986.

6. Massier, P., "The Need for Expanded Exploration of Matter-Antimatter Annihilation for Propulsion Applications," J. Br. Interplanetary Soc., 35 (9), 1982, pp. $387-$ 390.

7. Forward, R.L., "Antimatter Propulsion," J. Br. Interplanetary Soc., 35 (9), 1982. pp. 391-395.

8. Cassenti, B.N., "Design Considerations for Relativistic Antimatter Rockets," J. Br. Interplanetary Soc., 35 (9), 1982, pp. 396-404.

9. Morgan, D.L., "Concepts for the Design of an Antimatter Annihilation Rocket," J. Br. Interplanetary Soc., 35 (9), 1982, pp. 396-404.

10. Cassenti, B.N., "Antimatter Propulsion for OTV Applications," J. Propulsion and Power, 1 (2), 1985, pp. 143-149.

11. Vulpetti, G., "Antimatter Propulsion for Space Exploration," J. Br. Interplanetary Soc., 39 (9), 1986, pp. 391-409.

12. Forward, R.L., Advanced Space Propulsion Study: Antiproton and Beamed Power Propulsion, AFAL-TR87-070, Hughes Research Laboratories, Malibu, CA, Oct 1987.
13. Cassenti, B.N., "Energy Transfer in Antiproton Annihilation Rockets," in Antiproton Science and Technology, B.W. Augenstein et al., eds., World Scientific, Singapore, 1988, pp. 574-602.

14. Cassenti, B.N., "Conceptual Designs for Antiproton Propulsion Systems," AIAA Paper No. 89-2333, presented at the 25th Joint Propulsion Conference, Monterey, CA, Jul 10-12, 1989

15. Vulpetti, G., and Pecchioli, M., "Considerations About the Specific Impulse of an Antimatter-Based Thermal Engine," J. Propulsion and Power, 5 (5), 1989, pp. 591-595.

16. Howe, S.D., and Metzger, J.D., "Antiproton-Based Propulsion Concepts and Potential Impact on a Manned Mars Mission," J. Propulsion and Power, 5 (3), 1989, pp. 295-300.

17. Forward, R.L., 21st Century Space Propulsion Study, AL-TR-90-030, Forward Unlimited, Malibu, CA, Oct 1990.

18. Tarpley, C., Lewis, M.J., and Kothari, A.P., "Safety Issues is SSTO Spacecraft Powered by Antimatter Rocket Engines," AIAA Paper No. 90-2365, 26th Joint Propulsion Conference, Orlando, FL, Jul 16-18, 1990.

19. Cassenti, B.N., "High Specific Impulse Antimatter Rockets," AIAA Paper No. 91-2548, presented at the 27th Joint Propulsion Conference, Sacramento, CA, June 24-26, 1991.

20. Forward, R.L., and Davis, J., Mirror Matter: Pioneering Antimatter Physics, J. Wiley \& Sons, NY, NY, 1988.

21. LaPointe, M.R., Antiproton Annihilation Propulsion Using Magnetically Confined Plasma Engines, Dissertation, U. New Mexico, Dept. Chemical and Nuclear Engineering, Albuquerque, NM, 1989.

22. LaPointe, M.R., "Antiproton Powered Propulsion with Magnetically Confined Plasma Engines," J. Propulsion and Power, 7 (5), pp. 749-759.

23. Callas, J.L., The Application of Monte Carlo Modeling to Matter-Antimatter Annihilation Propulsion Concepts, JPL D-6830, Jet Propulsion Laboratory, Pasadena, CA, Oct 1989. 
24. Huber, F., Antimatterie-Annihilationsantriebe fur Interplanetare Raumfahrtmissionen, Ph.D. Dissertation, Universitat Stuttgart, Institute fur Raumfahrtsysteme, 1994.

25. Lewis, R.A., Newton, R., Smith, G.A., Toothacker, W.S., and Kanzleiter, R.J., "An Antiproton Catalyst for Inertial Confinement Fusion Propulsion," AIAA Paper No. 90-2760, presented at the 26th Joint Propulsion Conference, Orlando, FL, Jul 16-18, 1990.

26. Lewis, R.A., Smith, G.A., Toothacker, W.S., Kanzleiter, R.J., Surratt, M.S., Higman, K.I., and Newton, R.J., "An Antiproton Driver for Inertial Confinement Fusion Propulsion," AIAA Paper No. 91-3618, presented at AIAA/NASA/OAI Conf. on Advanced SEI Technologies, Cleveland, OH, Sep 4-6, 1991.

27. Schmidt, G. R., Gerrish, H. P., Martin, J. J., Smith, G. A., and Meyer, K. J., "Antimatter Requirements and Energy Costs for Near-Term Propulsion Applications," J. Propulsion and Power, 16 (5), Sep-Oct 2000, pp. 923-928.

28. Chapline, G. "Antimatter Breeders," J. Br. Interplanetary Soc., 35 (9), pp. 423-424, 1982.

29. Cassenti, B.N., "Concepts for the Efficient Production and Storage of Antimatter," AIAA-93-2031, presented at the 29th Joint Propulsion Conference, Monterey, CA, Jun 28-30, 1993.

30. Hora, H., "Estimates of the Efficient Production of Antihydrogen by Lasers of Very High Intensities," $O p$ toElectronics, 5, 1973, pp. 491-501.

31. Crowe, E.G., "Laser Induced Pair Production as a Matter-Antimatter Source," J. Br. Interplanetary Soc., 36, 1983, pp. 507-508.
32. Haloulakos, V., and Ayotte, A., "The Prospects for Space-Based Antimatter Production," AIAA Paper 9I1987, presented at the 27th Joint Propulsion Conference, Sacramento, CA, Jun 24-26, 1991.

33. Dirac, P.A.M., Directions in Physics, John Wiley \& Sons, New York, NY, 1978, pp. 1-37, 55-70.

34. Greiner, W., Relativistic Quantum Mechanics: Wave Equations, Springer-Verlag, Inc., Berlin, 1994, pp. 261-267.

35. Mostepanenko, V.M. and N.N. Trunov, The Casimir Effect and its Applications, Oxford Science Publications, Clarendon Press, Oxford, 1997.

36. Weinberg, S., The Quantum Theory of Fields, Vol. 1, Cambridge University Press, 1995, pp. 578-593.

37. Forward, R.L., Mass Modification Experiment Definition Study, PL-TR-96-3004, Forward Unlimited, Malibu, CA, Feb 1996.

38. Boyer, T.H., "The Classical Vacuum," Sci. Am., 253 (3), Aug 1985, pp. 70-78.

39. Milonni, P.W., The Quantum Vacuum: An Introduction to Quantum Electronics, Academic Press, New York, NY, 1994.

40. LaPointe, M. R., Antimatter Production at a Potential Boundary, NASA SBIR Phase I Final Report, Contract NAS8-98109, prepared for the NASA Marshall Space Flight Center, Huntsville, AL, Nov 1998. 
Davis Highway, Suite 1204, Arlington, VA 22202-4302, and to the Otfice of Management and Budget, Paperwork Reduction Project (0704-0188), Washington, DC 20503.

\begin{tabular}{|c|c|r|}
\hline $\begin{array}{c}\text { 1. AGENCY USE ONLY (Leave blank) } \\
\text { August 2001 }\end{array}$ & $\begin{array}{r}\text { 3. REPORT DATE } \\
\text { AuPORT TYPE AND DATES COVERED }\end{array}$ \\
Final Contractor Report
\end{tabular}

4. TITLE AND SUBTITLE

Antimatter Production at a Potential Boundary

6. AUTHOR(S)

Michael R. LaPointe
WU-755-B4-07-00

NCC $3-860$

NAS8-98109 SBIR

8. PERFORMING ORGANIZATION REPORT NUMBER

E-12962

Ohio Aerospace Institute

22800 Cedar Point Road

Brook Park, Ohio 44142

10. SPONSORING/MONITORING AGENCY REPORT NUMBER

National Aeronautics and Space Administration

Washington, DC 20546-000I

NASA CR - 2001-211116

AIAA-2001-3361

\section{SUPPLEMENTARY NOTES}

Prepared for the 37th Joint Propulsion Conference and Exhibit cosponsored by the AIAA, ASME, SAE, and ASEE, Salt Lake City, Utah, July 8-11, 2001. Project Manager, Dhanireddy Reddy, Power and On-Board Propulsion Technology Division, NASA Glenn Research Center, organization code 5430, 216-433-8133.

12a. DISTRIBUTION/AVAILABILITY STATEMENT 12b. DISTRIBUTION CODE

Unclassified - Unlimited

Subject Category: 73

Distribution: Nonstandard

Available electronically at hitp://gltrs, grc,nasa,gov/GLTRS

This publication is available from the NASA Center for AeroSpace Information, 301-621-0390.

13. ABSTRACT (Maximum 200 words)

Current antiproton production techniques rely on high-energy collisions between beam particles and target nuclei to produce particle and antiparticle pairs, but inherently low production and capture efficiencies render these techniques impractical for the cost-effective production of antimatter for space propulsion and other commercial applications. Based on Dirac's theory of the vacuum field, a new antimatter production concept is proposed in which particleantiparticle pairs are created at the boundary of a steep potential step formed by the suppression of the local vacuum fields. Current antimatter production techniques are reviewed, followed by a description of Dirac's relativistic quantum theory of the vacuum state and corresponding solutions for particle tunneling and reflection from a potential barrier. The use of the Casimir effect to suppress local vacuum fields is presented as a possible technique for generating the sharp potential gradients required for particle-antiparticle pair creation.

\section{SUBJECT TERMS}

Antimatter; Antiparticles; Antiprotons; Propulsion; Matter-antimatter propulsion 17

\begin{tabular}{|c|c|c|}
\hline $\begin{array}{c}\text { 17. SECURITY CLASSIFICATION } \\
\text { OF REPORT } \\
\text { Unclassified }\end{array}$ & $\begin{array}{c}\text { 18. SECURITY CLASSIFICATION } \\
\text { OF THIS PAGE } \\
\text { Unclassified }\end{array}$ & $\begin{array}{c}\text { 19. SECURITY CLASSIFICATION } \\
\text { OF ABSTRACT } \\
\text { Unclassified }\end{array}$ \\
\hline
\end{tabular}

